\title{
Orbifold Cohomology as Periodic Cyclic Homology
}

\author{
Vladimir Baranovsky
}

June 22, 2002

\begin{abstract}
It known from the work of Feigin-Tsygan, Weibel and Keller that the cohomology groups of a smooth complex variety $X$ can be recovered from (roughly speaking) its derived category of coherent sheaves. In this paper we show that for a finite group $G$ acting on $X$ the same procedure applied to $G$-equivariant sheaves gives the orbifold cohomology of $X / G$.

As an application, in some cases we are able to obtain simple proofs of an additive isomorphism between the orbifold cohomology of $X / G$ and the usual cohomology of its crepant resolution (the equality of Euler and Hodge numbers was obtained earlier by various authors). We also state some conjectures on the product structures, as well as the singular case; and a connection with a recent work by Kawamata.
\end{abstract}

\section{Introduction}

Let $X$ be a smooth variety over a field $k$ (for simplicity we assume in this introduction that $k=\mathbb{C}$ ) and $G$ be a finite group acting on $X$. If the quotient variety $X / G$ is Gorenstein (i.e. the canonical class is a Cartier divisor) and $\pi: Y \rightarrow X / G$ is a crepant resolution of singularities then Ruan's Cohomological Crepant Resolution Conjecture (which we call Cohomological Conjecture for short) states, as a particular case, that the cohomology groups $H^{*}(Y, \mathbb{C})$ should be isomorphic to the orbifold cohomology

$$
H_{\text {orb }}^{*}(X / G, \mathbb{C})=\left(\bigoplus_{g \in G} H^{*}\left(X^{g}, \mathbb{C}\right)\right)_{G}
$$

where $(\ldots)_{G}$ denotes the coinvariants, $X^{g}$ is the fixed point set, and $G$ acts on the above direct sum by conjugating $g$. This definition of $H_{\text {orb }}^{*}(X, \mathbb{C})$ is slightly different from the usual one, see Section 3 of $[\mathbb{R u}]$, but equivalent to it. Moreover, Ruan has introduced product structures on $H^{*}(Y, \mathbb{C})$ (a deformation of the usual product using the rational curves contracted by $\pi$ ) and $H_{\text {orb }}^{*}(X / G, \mathbb{C})$, loc. cit., and the Cohomological Conjecture states that $H^{*}(Y, \mathbb{C}) \simeq H_{\text {orb }}^{*}(X / G, \mathbb{C})$ is actually a ring isomorphism. On the level of Betti (or Hodge) numbers this conjecture was recently proved by Lupercio-Poddar and Yasuda, see [Y]. However, with the approach used in the proof (motivic integration) it is not clear how to identify the actual cohomology groups with their product structures. In this paper we will try to outline a different approach to the Cohomological Crepant Resolution Conjecture and show that it is in fact a consequence of a Categorical Resolution Conjecture stated (in a form and under a name slightly different from ours) by Kawamata in [Ka]. We hope that the categorical approach will allow to interpret the product structures. Besides, the author believes that it would be very important to establish a link between the categorical and the motivic integration approach; relating the derived category of sheaves to the space of arcs (or possibly the space of formal loops of Kapranov-Vasserot).

For simplicity we only work with global quotients $X / G$ but all statements can be made (and, hopefully, proved) for general smooth Deligne-Mumford stacks and categories of sheaves twisted by a gerbe.

In more detail: we would like to show that the above Cohomological Conjecture follows from an equivalence of two derived categories: the bounded derived category $D^{b}(Y)$ of coherent sheaves on $Y$ and the 
bounded derived category $D_{G}^{b}(X)$ of $G$-equivariant sheaves on $X$ (i.e. sheaves on the quotient stack $[X / G]$ ). Thus, a possible proof of the Cohomological Conjecture could consist of three steps:

(1) Prove an equivalence of derived categories $D^{b}(Y) \rightarrow D_{G}^{b}(X)$ (Categorical Resolution Conjecture see end of Section 5).

(2) Recover an isomorphism of cohomology groups $H^{*}(Y, \mathbb{C}) \rightarrow H_{\text {orb }}^{*}(X / G, \mathbb{C})$ from the above equivalence.

(3) Identify the two product structures.

In this paper we mostly deal with the second step. As for the other two, we note that (1) is known in some cases due to the work of Bridgeland-King-Reid and Kawamata (see [BKR], [Ka] and Section 4 of this paper); while the orbifold product in (3) seems to arise from the convolution product of sheaves (see Section 5).

To deal with (2) one needs a construction which recovers the (orbifold) cohomology ring from the (equivariant) derived category. In a sense, we are using some additional structure: the derived category should "remember" that it was obtained as a quotient of two DG-categories forming a localization pair, see Section 2.4 of [K1]. However, it follows from a result of Orlov [Or] that fully faithful exact functor between the (non-equivariant) derived categories automatically preserves this additional structure; and the same holds for equivalences of equivariant derived categories (in the case of a finite group action).

In the non-equivariant case a construction recovering the cohomology groups follows from the work of Feigin-Tsygan, Weibel and Keller. In fact, for any exact category $\mathcal{A}$, such as the category $V$ ect $(Y)$ of vector bundles on $Y$ or the category $V \operatorname{ect}_{G}(X)$ of $G$-equivariant vector bundles on $X$, Keller constructs in [K1] a mixed complex $C(\mathcal{A})$ which leads to a family of homology theories $H C \bullet(\mathcal{A}, W)$ depending on a graded $k[u]$-module $W$ (usually taken to be of finite projective dimension). We "recall" the relevant definitions in Section 2. Two properties make this construction very attractive in our setup:

- When $\mathcal{A}$ is the category of vector bundles on $Y, k=\mathbb{C}$ and $W=k\left[u, u^{-1}\right]$, the above homology group $H C_{0}(\mathcal{A}, W)$ (resp. $\left.H C_{1}(\mathcal{A}, W)\right)$ can be identified with $H^{\text {even }}(Y, \mathbb{C})$ (resp. $H^{\text {odd }}(Y, \mathbb{C})$ ). Note that multiplication by $u$ gives an isomorphism $H C_{i}(\mathcal{A}, W) \simeq H C_{i+2}(\mathcal{A}, W)$.

- For any $W$ the homology theory $H C \cdot(\mathcal{A}, W)$ is invariant with respect to equivalences of derived categories coming from functors between localization pairs.

To formulate our results, recall that $G$ acts on $\coprod_{g \in G} X^{g}: h \in G$ sends $x \in X^{g}$ to $h x \in X^{h g h^{-1}}$. This action is inherited by $\bigoplus_{g \in G} H C \cdot\left(\operatorname{Vect}\left(X^{g}\right), W\right)$.

Theorem 1 Let $G$ be a finite group acting on a smooth quasiprojective variety $X$ over a field $k$ of characteristic not dividing $|G|$. For any graded $k[u]$-module $W$ of finite projective dimension there exists an isomorphism functorial with respect to pullbacks under G-equivariant maps:

$$
\psi_{X}: H C_{\bullet}\left(\operatorname{Vect}_{G}(X), W\right) \simeq\left(\bigoplus_{g \in G} H C \bullet\left(V e c t\left(X^{g}\right), W\right)\right)_{G}
$$

where $(. . .)_{G}$ denotes the coinvariants.

In the $C^{\infty}$-manifold or $C^{\infty}$-etale groupoid setting this result (formulated in terms of modules over smooth functions rather than categories) has a long history. First Feigin-Tsygan, see [FT], constructed a spectral sequence computing cyclic homology of a general crossed product algebra, which was later reformulated by Getzler-Jones, see [G]2]; and also [AK] for more general crossed products by Hopf algebras. When the crossed product algebra comes from functions on a smooth manifold the $E_{2}$ term of this spectral sequence can be interpreted in terms of fixed point submanifolds: this result was announced in [Br] and the first published proof appears in $[B N]$. Later it was generalized in $[\mathrm{Cr}]$. The case when $G$ is a Lie group was studied by Nistor in [N]; later Block and Getzler have related the corresponding crossed product cyclic 
homology groups to equivariant differential forms and fixed points, see [BG]. We also mention a closely related computation of $G$-equivariant topological $K$-theory by G. Segal, see [HH]; and its algebraic $K$-theory counterparts [V] $[$ [T] $]$.

In Section 3 we adapt the proofs in [BG] and [G]2] to fit our case of categories and rings of regular functions. Note that the proof of $\llbracket \mathbb{V}]$ cannot be applied in our case due to the failure of devissage, see Example 1.11 in [K1].

In those cases when the derived equivalence $D^{b}(Y) \rightarrow D_{G}^{b}(X)$ is known, we get an isomorphism between $H C \cdot(V e c t(Y), W)$ and the right hand side expression in Theorem 1, obtaining a slightly generalized version of the Cohomological Conjecture (in general the cyclic homology groups do not satisfy the long exact sequence hence even on the level of dimensions the equality cannot be derived using motivic measures and motivic integration). This is the second main result of this paper (see Corollary 12).

We expect that, in order to identify the product structures in step (3) above, one should modify $\psi_{X}$ to make it compatible with pushforwards under $G$-equivariant closed embeddings, rather than pullbacks (compare with Lemmas 4.2 and 4.3 in [प]

We note here that for a general algebraic group $G$ the equivariant derived category $D_{G}^{b}(X)$ is defined not by taking complexes of $G$-equivariant sheaves but by a more delicate localization procedure, see [BL]. One can expect that the corresponding cyclic homology groups satisfy nice properties, for example similar to those proved in [B]].

This paper is organized as follows. Section 2 gives some basic information of cyclic homology groups of exact categories. In Section 3 we prove Theorem in. In Section 4 we show how an equivalence of derived categories implies equality of (orbifold) cohomology groups and also give some examples in which this equivalence is known. Finally, in Section 5 we give a conjecture about the singular case and a conjecture on how the orbifold cohomology product can be recovered from the convolution product in the derived category.

Acknowledgements. The present work was motivated by a lecture of Y. Ruan on orbifold cohomology given by him at Caltech; and the two beautiful papers by B. Keller [K1], [K2] on cyclic homology of exact categories. The author is grateful to both of them for providing this inspiration.

\section{Generalities on Mixed Complexes}

Recall, cf [W1] that a mixed complex over a commutative ring $k$ is a sequence of $k$-modules $\left\{C_{m}: m \geq 0\right\}$ with two families of morphisms $b: C_{m} \rightarrow C_{m-1}$ and $B: C_{m} \rightarrow C_{m+1}$ satisfying $b^{2}=B^{2}=B b+b B=0$. To any such mixed complex one can apply the following formalism (see [GJ1]): let $W$ be a graded module over the polynomial ring $k[u]$, where $\operatorname{deg}(u)=-2$ (in practice it is always assumed that $W$ has finite homological dimension). Then one can form a complex $C[[u]] \otimes_{k[u]} W$ with a differential $b+u B$ and compute its cohomology groups, to be denoted by $H C \bullet(C, W)$. The following are important examples:

- $W=k[u] / u k[u]$ gives the Hochschild homology $H H_{\bullet}(C)$

- $W=k\left[u, u^{-1}\right] / u k[u]$ gives cyclic homology $H C \bullet(C)$

- $W=k\left[u, u^{-1}\right]$ gives periodic cyclic homology $H P \bullet(C)$

- $W=k[u]$ gives negative cyclic homology $H N_{\bullet}(C)$ (sometimes also denoted by $H C_{\bullet}^{-}(C)$ ).

The following lemma shows that for some purposes it suffices to consider only the first case.

Lemma 2 Let $f:(C, b, B) \rightarrow\left(C^{\prime}, b^{\prime}, B^{\prime}\right)$ be a map of mixed complexes such that $f$ induces an isomorphism $H(C, b) \rightarrow H\left(C^{\prime}, b^{\prime}\right)$. Then for any coefficients $W$ of finite projective dimension over $k[u]$,

$$
f: \quad H_{\bullet}\left(C[[u]] \otimes_{k[u]} W, b+u B\right) \rightarrow H_{\bullet}\left(C^{\prime}[[u]] \otimes_{k[u]} W, b^{\prime}+u B^{\prime}\right)
$$

is an isomorphism. 
Proof. See Proposition 2.4 in [GJ1].

This lemma justifies the following point of view on mixed complexes, see [K1], Section 1.2. Let $\Lambda$ be the DG-algebra generated by an indeterminate $\varepsilon$ of chain degree 1 with $\varepsilon^{2}=0$ and $d \varepsilon=0$. Then a mixed complex may be identified with a left $\Lambda$-module whose underlying DG $k$-module is $(C, b)$ and where $\varepsilon$ acts by $B$. Moreover, if we are interested only in the resulting homology groups (as is the case in this paper), we can view a mixed complex as an object in the derived category of the DG algebra $\Lambda$.

In what follows we will need a definition of the mapping cone over a map $f: C \rightarrow C^{\prime}$ of mixed complexes. It is given by the mixed complex

$$
\left(C^{\prime} \oplus C[1],\left[\begin{array}{cc}
b_{C^{\prime}} & f \\
0 & -b_{C}
\end{array}\right],\left[\begin{array}{cc}
B_{C^{\prime}} & 0 \\
0 & -B_{C}
\end{array}\right]\right)
$$

Now we briefly recall Keller's construction of the mixed complex $C(\mathcal{A})$ of an exact category $\mathcal{A}$ over a field $k$ (actually in [K1] the complex is defined for any commutative ring $k$ but the general definition is somewhat more involved). Starting from $\mathcal{A}$ one can construct its category $\mathcal{C}^{b} \mathcal{A}$ of all bounded complexes over $\mathcal{A}$ and the category $\mathcal{A} c^{b} \mathcal{A}$ of bounded acyclic complexes over $\mathcal{A}$. Both $\mathcal{C}^{b} \mathcal{A}$ and $\mathcal{A} c^{b} \mathcal{A}$ are $D G$ categories, i.e. for any pair of objects $X, Y$ the group $\operatorname{Hom}(X, Y)$ is $\mathbb{Z}$-graded (by degree of a map) with a differential, which satisfies some natural axioms (see [K3])).

For any small DG category $\mathcal{B}$ over a field $k$ Keller constructs a mixed complex as follows. Denote for notational convenience $\operatorname{Hom}_{\mathcal{B}}(X, Y)$ by $(X \rightarrow Y)$ and consider for each $n \in \mathbb{N}$ a vector space

$$
C_{n}(\mathcal{B})=\bigoplus\left(B_{0} \rightarrow B_{1}\right) \otimes\left(B_{1} \rightarrow B_{2}\right) \otimes \ldots \otimes\left(B_{n-1} \rightarrow B_{n}\right) \otimes\left(B_{n} \rightarrow B_{0}\right)
$$

where the sum runs over all sequences $B_{0}, \ldots, B_{n}$ of objects of $\mathcal{B}$. The face maps

$$
d_{i}\left(f_{0}, \ldots, f_{i}, f_{i+1}, \ldots, f_{n}\right)= \begin{cases}\left(f_{0}, \ldots, f_{i} f_{i+1}, \ldots, f_{n}\right) & \text { if } 0 \leq i \leq n-1 \\ (-1)^{n+\sigma}\left(f_{n} f_{0}, \ldots, f_{n-1}\right) & \text { if } i=n\end{cases}
$$

(where $\left.\sigma=\operatorname{deg} f_{n} \cdot\left(\operatorname{deg} f_{0}+\ldots+\operatorname{deg} f_{n-1}\right)\right)$; together with the degeneracy maps

$$
s_{i}\left(f_{0}, \ldots, f_{i}, f_{i+1}, \ldots, f_{n}\right)=\left(f_{0}, \ldots, f_{i}, i d_{B_{i}}, f_{i+1}, \ldots, f_{n}\right) \quad i=0, \ldots, n
$$

and the cyclic operator

$$
t_{n}\left(f_{0}, \ldots, f_{n}\right)=(-1)^{n+\sigma}\left(f_{n}, f_{0}, f_{1}, \ldots, f_{n-1}\right),
$$

define a mixed complex $(C(\mathcal{B}), b,(1-t) s N)$ as in [G]2], Section 2. Note that, unlike in [K1], we write $f g$ for a composition of $f: A \rightarrow B$ and $g: B \rightarrow C$ instead of the usual $g f$. This non-traditional notation will allow us later to match our formulas with those of Getzler-Jones in [G]2].

Next, for any DG subcategory $\mathcal{C} \subset \mathcal{B}$ one has a mixed complex

$$
C(\mathcal{C}, \mathcal{B})=\operatorname{Cone}(C(\mathcal{C}) \rightarrow C(\mathcal{B})) .
$$

We will always use this definition when $(\mathcal{C}, \mathcal{B})$ is a localization pair in the sense of [K1], Section 2.4. Every such localization pair leads to a triangulated category $\mathcal{T}=\mathcal{B} / \mathcal{C}$ associated to it. For example, the pair $\left(\mathcal{A} c^{b} \mathcal{A}, \mathcal{C}^{b} \mathcal{A}\right)$ gives rise to the derived category $\mathcal{T}$ of $\mathcal{A}$. One of the main properties of the mixed complex $C(\mathcal{C}, \mathcal{B})$ is expressed in the following proposition (see Theorem 2.4(a) in [K1]):

Proposition 3 Let $(\mathcal{C}, \mathcal{B})$ and $\left(\mathcal{C}^{\prime}, \mathcal{B}^{\prime}\right)$ be two localization pairs and $\mathcal{T}, \mathcal{T}^{\prime}$ their derived categories. If $F: \mathcal{B} \rightarrow \mathcal{B}^{\prime}$ is an exact functor which takes $\mathcal{C}$ to $\mathcal{C}^{\prime}$ and induces and equivalence up to factors $\mathcal{T} \rightarrow \mathcal{T}^{\prime}$ (cf. 1.5 in [K1]), then $F$ induces an isomorphism $C(\mathcal{C}, \mathcal{B}) \rightarrow C\left(\mathcal{C}^{\prime}, \mathcal{B}^{\prime}\right)$ in the derived category of $\Lambda$.

If $\mathcal{A}$ is an exact category then its mixed complex is defined as $C\left(\mathcal{A} c^{b} \mathcal{A}, \mathcal{C}^{b} \mathcal{A}\right)$. We will write $\mathcal{A} c^{b}(X)$ (resp. $\left.\mathcal{C}^{b}(X)\right)$ instead of $\mathcal{A} c^{b} V e c t(X)$ (resp. $\left.\mathcal{C}^{b} V e c t(X)\right)$ and similarly for $\mathcal{A} c_{G}^{b}(X)$ and $\mathcal{C}_{G}^{b}(X)$. Define $C(X)=C\left(\mathcal{A} c^{b}(X), \mathcal{C}^{b}(X)\right) ; C_{G}(X)=C\left(\mathcal{A} c_{G}^{b}(X), \mathcal{C}_{G}^{b}(X)\right)$. 


\section{Mixed Complex for a Finite Group Action}

In this section we will prove Theorem 1. In view of Lemma 2 and the subsequent remarks, it suffices to prove the following proposition:

Proposition 4 Let $G$ be a finite group acting on a smooth quasiprojective variety $X$ over a field $k$ of characteristic not dividing $|G|$. There exists a quasiisomorphism in the derived category of $\Lambda$ :

$$
\psi_{X}: C_{G}(X) \rightarrow\left(\bigoplus_{g \in G} C\left(X^{g}\right)\right)_{G}
$$

which is functorial with respect to pullbacks under G-equivariant maps of smooth varieties $f: Y \rightarrow X$.

The proof of the above proposition will be carried out in several steps: first we replace $V e c t_{G}(X)$ by a categorical analogue of a crossed product ring $A \rtimes G$ and construct a functorial map of objects in the derived category (Steps 1 and 2). Once this map is constructed, we use Mayer-Vietoris, Luna's Fundamental Lemma and etale descent of Weibel-Geller to reduce to the case when $X$ is a vector space with a linear action of $G$; and conclude the argument using exactness of a Koszul complex (Steps 3-6). Our proof is an adaptation of the argument in [BG] from the differentiable to the algebraic situation.

Step 1: Change of categories.

Note that the action of $G$ on $X$ is inherited by the categories $\operatorname{Vect}_{G}(X), \mathcal{C}^{b}(X)$ and $\mathcal{A} c^{b}(X)$ : for any $g \in G$ and any bundle (or a complex of bundles) $\mathcal{F}$ we can consider $g \mathcal{F}:=\left(g^{-1}\right)^{*} \mathcal{F}$ and for any $\psi: \mathcal{F} \rightarrow \mathcal{G}$ we have $g(\psi): g \mathcal{F} \rightarrow g \mathcal{G}$.

For any $\mathcal{F}$ consider the object $\widetilde{\mathcal{F}}=\bigoplus_{g \in G} g \mathcal{F}$ with its natural $G$-equivariant structure. Then each object $\mathcal{H}$ in $\mathcal{C}^{b} \operatorname{Vect}_{G}(X)$ is isomorphic to a direct factor of some $\widetilde{\mathcal{F}}$. In fact, take $\mathcal{F}=\mathcal{H}$ viewed as an object in $\mathcal{C}^{b} \operatorname{Vect}(X)$. Then the $G$-equivariant structure on $\mathcal{H}$ defines isomorphisms $i_{g}: \mathcal{H} \rightarrow g \mathcal{H}$ for all $g \in G$. Now consider the $G$-equivariant maps

$$
\mathcal{H} \stackrel{a}{\longrightarrow} \widetilde{\mathcal{H}}=\bigoplus_{g \in G} g \mathcal{H} \stackrel{b}{\longrightarrow} \mathcal{H}
$$

where $a$ is given by a direct sum of $i_{g}$ and $b=\frac{1}{|G|} \sum i_{g}$. Since $b \circ a=i d_{\mathcal{H}}, \mathcal{H}$ splits off as a direct factor of $\widetilde{\mathcal{H}}$.

Denote by $\mathcal{C}^{b}(X) \rtimes G$, resp. $\mathcal{A} c^{b}(X) \rtimes G$ the full subcategory of $\mathcal{C}_{G}^{b}(X)$, resp. $\mathcal{A} c_{G}^{b}(X)$, formed by all $\widetilde{\mathcal{F}}$. Since $\mathcal{C}^{b}(X) \rtimes G$ and $\mathcal{A} c^{b}(X) \rtimes G$ are closed under degree-wise split extensions and shifts, both are exact DG categories (by Example 2.2 (a) in [K1]). The above argument shows that the natural embedding $\mathcal{C}^{b}(X) \rtimes G \rightarrow \mathcal{C}_{G}^{b}(X)$ induces an equivalence up to factors of associated derived categories. Now Theorem 2.4 (a) in [K1] gives

Proposition 5 There exists an isomorphism in the mixed derived category

$$
C\left(\mathcal{A} c^{b}(X) \rtimes G, \mathcal{C}^{b}(X) \rtimes G\right) \rightarrow C_{G}(X)
$$

which is functorial with respect to G-equivariant pullbacks of vector bundles.

Step 2: Construction of the Quasiisomorphism. 
To proceed further, we take a closer look at $\mathcal{C}^{b}(X) \rtimes G$. The objects in this category can be identified with objects in $\mathcal{C}^{b}(V e c t(X))$ while the morphisms are given by

$$
(\mathcal{F} \rightarrow \mathcal{G})_{\mathcal{C}^{b}(X) \rtimes G}=\bigoplus_{g \in G}(\mathcal{F} \rightarrow g \mathcal{G})_{\mathcal{C}^{b}(X)}
$$

(the expression on the right obviously coincides with all $G$-equivariant morphisms from $\widetilde{\mathcal{F}}$ to $\widetilde{\mathcal{G}}$ ). If we denote by $\psi \cdot g$ and $\varphi \cdot h$ the components of $(\mathcal{F} \rightarrow \mathcal{G})_{\mathcal{C}^{b}(X) \rtimes G}$ and $(\mathcal{G} \rightarrow \mathcal{H})_{\mathcal{C}^{b}(X) \rtimes G}$ living in $(\mathcal{F} \rightarrow g \mathcal{G})_{\mathcal{C}^{b}(X)}$ and $(\mathcal{G} \rightarrow h \mathcal{H})_{\mathcal{C}^{b}(X)}$, respectively, then the composition of $\psi \cdot g$ and $\varphi \cdot h$ is given by

$$
\psi g(\varphi) \cdot g h: \mathcal{F} \rightarrow g \mathcal{G} \rightarrow g h \mathcal{H}
$$

Now define the map

$$
C\left(\mathcal{C}^{b}(X) \rtimes G\right) \rightarrow\left(\bigoplus_{g \in G} C\left(\mathcal{C}^{b}\left(X^{g}\right)\right)\right)_{G}
$$

by sending $\left(\varphi_{0} \cdot g_{0}, \varphi_{1} \cdot g_{1}, \ldots, \varphi_{n} \cdot g_{n}\right)$ to $\left(\varphi_{\left.0\right|_{X} g}, g_{0}\left(\varphi_{1}\right)_{\left.\right|_{X g}}, g_{0} g_{1}\left(\varphi_{2}\right)_{\left.\right|_{X g}}, \ldots,\left(g_{0} \ldots g_{n-1}\right)\left(\varphi_{n}\right)_{\left.\right|_{X g} g}\right)$ where $g=g_{0} \ldots g_{n}$. Note that only the map to the coinvariants is indeed a map of mixed complexes since the individual maps $C\left(\mathcal{C}^{b}(X) \rtimes G\right) \rightarrow C\left(\mathcal{C}^{b}\left(X^{g}\right)\right)$ do not respect the last face map $d_{n}$ and the cyclic operator $t_{n}$. Since the above morphism obviously sends $C\left(\mathcal{A} c^{b}(X) \rtimes G\right)$ to $\left(\bigoplus_{g \in G} C\left(\mathcal{A} c^{b}\left(X^{g}\right)\right)\right)_{G}$ we obtain a morphism of mixed complexes

$$
C\left(\mathcal{A} c^{b}(X) \rtimes G, \mathcal{C}^{b}(X) \rtimes G\right) \rightarrow\left(\bigoplus_{g \in G} C\left(\mathcal{A} c^{b}\left(X^{g}\right), \mathcal{C}^{b}\left(X^{g}\right)\right)\right)_{G} .
$$

Composing this map with the inverse of the quasiisomorphism in Proposition 5 we obtain a morphism of objects in the derived category of $\Lambda$

$$
\psi_{X}: C_{G}(X) \rightarrow\left(\bigoplus_{g \in G} C\left(X^{g}\right)\right)_{G}
$$

which is functorial with respect to pullbacks under $G$-equivariant maps $f: Y \rightarrow X$.

Step 3: Mayer-Vietoris and Luna's Fundamental Lemma

Now we use a Mayer-Vietoris sequence to reduce to the case when $X$ is affine.

Proposition 6 Let $X$ be a quasiprojective scheme, $V, W \subset X$ two $G$-invariant open subschemes and $U=V \cap W$. There is a distinguished triangle in the mixed derived category category:

$$
C_{G}(X) \rightarrow C_{G}(V) \oplus C_{G}(W) \rightarrow C_{G}(U) \rightarrow C_{G}(X)[1]
$$

Outline of Proof. Most of the argument is identical to the non-equivariant case proved in Proposition 5.8 of [K2]. First one shows that for a quasiprojective scheme $X, C_{G}(X)$ is quasiisomorphic to the complex obtained from the category of $G$-equivariant perfect complexes (see Section 5.1 of [K2]]). Moreover, let $\mathcal{T}_{G}(X)$ be the derived category of $G$-equivariant perfect complexes and for any closed $Z \subset X$ by $\mathcal{T}_{G}(X$ on $Z$ ) the subcategory of complexes which are exact on the complement to $Z$. If $Z=X \backslash W$ and $j: V \rightarrow X$ is the open embedding, one shows that the lines of the diagram

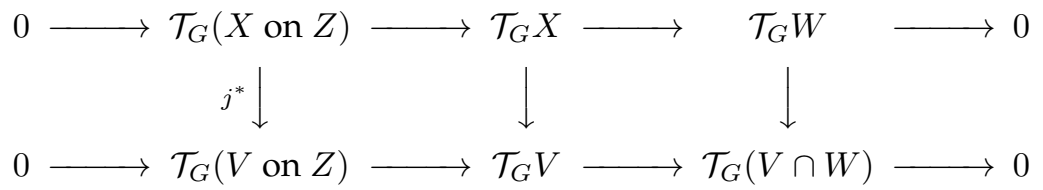


are exact up to factors and the functor $j^{*}$ is an equivalence up to factors (the proof in Sections 5.4 and 5.5 of [TT] may be repeated almost word-by-word; note also that working "up to factors" allows one to ignore all problems with non-surjectivity of $K_{G}^{0}(X) \rightarrow K_{G}^{0}(W)$ and so on, since for any complex $E$, the sheaf $E \oplus E[1]$ has zero class in $K$-theory). Finally, one applies Theorem 2.7 of [K1].

Alternatively, one can work with $\mathcal{C}^{b}(X) \rtimes G$ and deduce the above fact from the non-equivariant version ([K1], Proposition 5.8)

The above Mayer-Vietoris sequence shows that if $\psi_{U}, \psi_{V}$ and $\psi_{W}$ are quasiisomorphisms then the same holds for $\psi_{X}$ (of course, this is only possible by functoriality of $\psi_{X}$ applied to open embeddings). By induction on the number of elements in a $G$-invariant affine covering of $X$ we can assume that $X=S p e c A$ is affine. In this case we can reinterpret the map $\psi_{X}$ as follows. The group $G$ acts on the $k$-algebra $A$ and we can define $A \rtimes G$ to be the crossed product algebra $A \otimes k[G]$ with the product defined by

$$
\left(a_{1} \cdot g_{1}\right)\left(a_{2} \cdot g_{2}\right)=\left(a_{1} g_{1}\left(a_{2}\right)\right) \cdot g_{1} g_{2}
$$

Then the category $\operatorname{Vect}_{G}(X)$ is equivalent to the category of $A \rtimes G$-modules which are projective as $A$ modules. Since $G$ is finite, this is equivalent to being projective as $A \rtimes G$-modules.

Now consider the category dgfree $A \rtimes G$ of complexes of free $A \rtimes G$-modules. By Section 2.4 of [K1] the natural functor $d g$ free $A \rtimes G \rightarrow \mathcal{C}^{b} \operatorname{Vect}_{G}(X)$ induces a quasiisomorphism of mixed complexes

$$
C(0, \text { dgfree } A \rtimes G) \rightarrow C_{G}(X) .
$$

Moreover, if one considers $A \rtimes G$ as a subcategory of $d g$ free $A \rtimes G$ with one object (a free rank one module viewed as a complex in degree 0 ) then by Theorem 2.4 (a) of [K1] one gets a quasiisomorphism

$$
C(A \rtimes G) \rightarrow C(0, \text { dgfree } A \rtimes G)
$$

where the mixed complex $C(B)$ of any $k$-algebra $B$ is defined as in Section 2 (if one views $B$ as a category with one object). Thus, we obtain a chain of quasiisomorphisms

$$
C(A \rtimes G) \rightarrow C(0, \text { dgfree } A \rtimes G) \rightarrow C\left(\mathcal{A} c^{b}(X) \rtimes G, \mathcal{C}^{b}(X) \rtimes G\right) \rightarrow C_{G}(X)
$$

induced by embeddings of subcategories.

Similarly, if $g \in G$ and $J_{g} \subset A$ denotes the ideal of the fixed point set $X^{g} \subset X$ then we have a chain of quasiisomorphisms

$$
C\left(A / J_{g}\right) \rightarrow C\left(0, d g \text { free } A / J_{g}\right) \rightarrow C\left(X^{g}\right)
$$

Restricting the map $\psi_{X}$ constructed in the previous step, it suffices to prove that the following map is a quasiisomorphism in the derived category of $\Lambda$ :

$$
\psi_{A}: C(A \rtimes G) \rightarrow\left(\bigoplus_{g \in G} C\left(A / J_{g}\right)\right)_{G} ;
$$

where $\psi_{A}\left(a_{0} \cdot g_{0}, \ldots, a_{n} \cdot g_{n}\right)$ is the image of $\left(a_{0}, g_{0}\left(a_{1}\right), \ldots,\left(g_{0} \ldots g_{n-1}\right)\left(a_{n}\right)\right) \in A^{\otimes n+1}$ in $\left(A / J_{g}\right)^{\otimes n+1}=$ $C_{n}\left(A / J_{g}\right)$ and $g=g_{0} \ldots g_{n}$.

Eventually we will assume that $X$ is not only affine but has some additional properties. The following proposition is probably well-known but the author was unable to find a convenient reference.

Proposition 7 Let $G$ be a finite group acting on a smooth quasiprojective variety $X$ over a field $k$. Assume that $|G|$ is invertible in $k$. There exists a covering of $X$ by $G$-invariant affine open subsets $U_{1}, \ldots, U_{n}$ such that for any $i=1, \ldots, n$ there is a point $x_{i} \in U_{i}$ satisfying the following properties:

(a) the fixed point scheme $U_{i}^{g}$ is empty unless $g \in G_{x_{i}}$ (the stabilizer of $x_{i}$ ); 
(b) if $T_{x_{i}}$ is the tangent space to $U_{i}$ at $x_{i}$ with its natural $G_{x_{i}}$ action, then there exists a $G_{x_{i}}$-equivariant etale morphism $\varphi_{i}: U_{i} \rightarrow T_{x_{i}}$ such that for any subgroup $H \subset G_{x_{i}}$ the induced morphism $U_{i} / H \rightarrow T_{x_{i}} / H$ is etale and the diagram

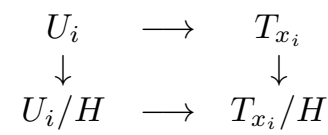

is Cartesian;

(c) for any $H \subset G_{x_{i}}$ the fixed point scheme $U_{i}^{H}$ is a scheme-theoretic preimage of $T_{x_{i}}^{H}$.

Proof. For any point $x \in X$ we can choose and open affine $G$-invariant neighborhood $U$ such that (a) holds. By Lemma 8.3 of [BR] there exists a $G_{x}$-equivariant map $\varphi: U \rightarrow T_{x}$ such that $\varphi(x)=0$ and $d \varphi(x)$ is equal to identity. Now by Theorem 6.2 of [BR] (Luna's Fundamental Lemma in finite characteristic) applied to the finite set of subgroups $H \in G_{x_{i}}$ we can shrink $U$ so that (b) holds as well.

For (c) let $U_{i}=\operatorname{Spec} B$ and $T_{x_{i}}=\operatorname{Spec} A$. Denote by $I_{A}$ the $A^{H}$-submodule of $A$ generated by elements $a-h(a), a \in A, h \in H$. Let also $J_{A}$ be the ideal in $A$ generated by $I_{A}$; then $J_{A}$ is the ideal of the fixed point scheme $T_{x_{i}}^{H}$. We use the similar notation $I_{B}, J_{B}$ for the objects corresponding to $B$. Since $B$ is flat over $A, J_{A} \otimes_{A} B$ is naturally an ideal in $B=A \otimes_{A} B$ and it suffices to prove that it coincides with $J_{B}$ (apriori we just have an inclusion $\left.J_{A} \otimes_{A} B \subset J_{B}\right)$. Note that since $|H|$ is invertible $A=I_{A} \oplus A^{H}$ as $A^{H}$-modules. Applying $\otimes_{A^{H}} B^{H}$ and using the fact that $B=A \otimes_{A^{H}} B^{H}$ by part (b) we conclude that $I_{B}=I_{A} \otimes_{A^{H}} B^{H}$ which implies $J_{B}=J_{A} \otimes_{A} B$ by definition of $J_{A}, J_{B}$.

Thus, later we may assume that $X$ is affine and there exists $x \in X$ such that (a), (b) and (c) above are satisfied.

Step 4: Eilenberg-Zilber Theorem

The mixed complex $C(A \rtimes G)$ was studied in a more general situation by Jones and Getzler [G]2]. In the next proposition we present their results (with some simplifications possible due to the fact that $|G|$ is invertible in $k$; also our isomorphism is more explicit than in [GJ2]).

To state the lemma we need to fix some notation. For any $g \in G$ consider the sequence of vector spaces $\left(A_{g}^{\natural}\right)_{n}=A^{\otimes n+1}$ together with the face, degeneracy and cyclic operators defined by the formulas similar to those in Section 2:

$$
\begin{gathered}
d_{i}\left(a_{0}, \ldots, a_{i}, a_{i+1}, \ldots, a_{n}\right)= \begin{cases}\left(a_{0}, \ldots, a_{i} a_{i+1}, \ldots, a_{n}\right) & \text { if } 0 \leq i \leq n-1 \\
\left(g^{-1}\left(a_{n}\right) a_{0}, \ldots, a_{n-1}\right) & \text { if } i=n\end{cases} \\
s_{i}\left(a_{0}, \ldots, a_{n}\right)=\left(a_{0}, \ldots, a_{i}, 1, a_{i+1}, \ldots, a_{n}\right) \quad i=0, \ldots, n \\
t_{n}\left(a_{0}, \ldots, a_{n}\right)=\left(g^{-1}\left(a_{n}\right), a_{0}, \ldots, a_{n-1}\right)
\end{gathered}
$$

Note that the operator $t_{n}$ does not satisfy the cyclic identity $t^{n+1}=1$. However, we can still construct a mixed complex from the spaces $A_{g}^{\natural}$ by considering a $G$-action on the direct sum $\bigoplus_{g \in G} A_{g}^{\natural}$ such that $h \in G$ sends $\left(a_{0}, \ldots, a_{n}\right) \in A_{g}^{\natural}$ to $\left(h\left(a_{0}\right), \ldots, h\left(a_{n}\right)\right) \in A_{h g h^{-1}}^{\natural}$. Then this $G$-action commutes with $d_{i}, s_{i}, t$ hence we obtain the face, degeneracy and cyclic operators on the quotient space $\left(\bigoplus_{g} A_{g}^{\natural}\right)_{G}$ of $G$-coinvariants. In this quotient space the operator $t_{n}$ does satisfy $t_{n}^{n+1}=1$ and we denote the resulting mixed complex by $C\left(\oplus_{g \in G} A_{g}^{\natural}\right)_{G}$.

Proposition 8 The map of mixed complexes

$$
\varphi_{A}: C(A \rtimes G) \rightarrow C\left(\bigoplus_{g \in G} A_{g}^{\natural}\right)_{G}
$$


defined by $\varphi\left(a_{0} \cdot g_{0}, \ldots a_{n} \cdot g_{n}\right)=\left(a_{0}, g_{0}\left(a_{1}\right), g_{0} g_{1}\left(a_{2}\right), \ldots, g_{0} \ldots g_{n-1}\left(a_{n}\right)\right) \in\left(A_{g}^{\natural}\right)_{n}$ with $g=g_{0} \ldots g_{n}$; is a quasiisomorphism in the derived category of $\Lambda$.

Proof. In [G]2] Getzler and Jones define a bi-graded object $A \natural G(p, q)=k\left[G^{p+1}\right] \otimes A^{\otimes q+1}$ with two families of face maps $d^{h}: A \natural G(p, q) \rightarrow A \natural G(p-1, q)$ (horizontal maps) and $d^{v}: A \llbracket G(p, q) \rightarrow A \natural G(p, q-1)$ (vertical maps), and similarly for degeneracies and cyclic operators (we will not use the precise definitions of these operators). These two families of operators give $A \curvearrowleft G(p, q)$ the structure of a cylindrical module, see [GJ2] before Proposition 1.1.

This cylindrical module has a total complex $\operatorname{Tot}_{n}(A, G)=\bigoplus_{p+q=n} A \natural G(p, q)$ and a diagonal complex $\Delta_{n}(A, G)=A \natural G(n, n)$, both being mixed complexes (see [G]2] for more details). The cylindrical module structure on $A \sharp G(p, q)$ is defined in such a way that $C(A \rtimes G)$ is isomorphic to the diagonal complex $\Delta(A, G)$ via the map

$$
\left(a_{0} \cdot g_{0}, \ldots, a_{n} \cdot g_{n}\right) \mapsto\left(g_{0}, \ldots, g_{n} \mid h_{0}^{-1} a_{0}, \ldots, h_{n}^{-1} a_{n}\right) \in A \natural G(n, n)=\Delta_{n}(A, G)
$$

where $h_{i}=g_{i} \ldots g_{n}$. Applying the Eilenberg-Zilber Theorem for paracyclic modules, see Theorem 3.1 in [GJ2], one gets an explicit quasiisomorphism

$$
A W: \Delta(A, G) \rightarrow \operatorname{Tot}(A, G)
$$

given by the Alexander-Whitney map defined in Section 8.5.2 of [W1]. We will only need one component of this map (with values in $A \npreceq G(0, n)$ ) which is given simply by $d_{1}^{h} \ldots d_{n}^{h}$, where $d_{i}^{h}: A \npreceq G(i, q) \rightarrow A \llbracket G(i-1, q)$ sends $\left(g_{0}, \ldots, g_{i} \mid a_{0}, \ldots, a_{q}\right)$ to $\left(g_{i} g_{0}, \ldots, g_{i-1} \mid g_{i}\left(a_{0}\right), \ldots, g_{i}\left(a_{0}\right)\right)$.

Now the transformation

$$
\left(g_{0}, \ldots, g_{p} \mid a_{0}, \ldots, a_{q}\right) \mapsto\left(g_{1}, \ldots, g_{p}\left|g_{0} g_{1} \ldots g_{p}\right| a_{0}, \ldots, a_{q}\right)
$$

identifies $A \llbracket G(p, q)$ with a cylindrical module the vertical maps of which are given by the above operators on $\bigoplus_{g \in G} A_{g}^{\natural}$ (and the index $g$ corresponds to $(\ldots|g| \ldots)$ in the above notation), while the rows can be identified with the standard homological complex of $G$ acting on $\bigoplus_{g \in G} A_{g}^{\natural}$. See Section 4 of [GJ2] for details. Since $|G|$ is invertible in $k$, the projection of $\operatorname{Tot}(A, G)$ onto its first column $A \curvearrowleft(0, \bullet)$ together with the projection to the coinvariants, gives a quasiisomorphism

$$
\operatorname{Tot}(A, G) \rightarrow C\left(\bigoplus_{g \in G} A_{g}^{\natural}\right)_{G} \cdot
$$

The fact that it is indeed a quasiisomorphism can be proved using the homotopy

$$
h: A \llbracket G(p, q) \rightarrow A \llbracket G(p+1, q) ; \quad\left(g_{1}, \ldots, g_{p}|g| a_{0} \ldots a_{q}\right) \mapsto \frac{1}{|G|} \sum_{g^{\prime} \in G}\left(g^{\prime}, g_{1}, \ldots, g_{p}|g| a_{0}, \ldots, a_{q}\right)
$$

The composition of (quasi)isomorphisms:

$$
C(A \rtimes G) \rightarrow \Delta(A, G) \rightarrow \operatorname{Tot}(A, G) \rightarrow C\left(\bigoplus_{g} A_{g}^{\natural}\right)_{G}
$$

is given by

$$
\left(a_{0} \cdot g_{0}, \ldots, a_{n} \cdot g_{n}\right) \rightarrow\left(g_{0}^{-1}\left(a_{0}\right), a_{1}, g_{1}\left(a_{2}\right), \ldots,\left(g_{1} \ldots g_{n-1}\right)\left(a_{n}\right)\right) \in\left(A_{g}^{\natural}\right)_{n} ; \quad g=g_{1} g_{2} \ldots g_{n} g_{0} .
$$

Note that this differs from the map in the statement of our proposition exactly be the action of $g_{0} \in G$. Since on the space of coinvariants $g_{0}$ acts by identity, this finishes the proof. 
Thus, we have reduced Proposition 4 to the claim that the map

$$
C\left(\bigoplus_{g \in G} A_{g}^{\natural}\right)_{G} \rightarrow\left(\bigoplus_{g \in G} C\left(A / J_{g}\right)\right)_{G}
$$

defined by the natural surjections $\left(A_{g}^{\natural}\right)_{n} \rightarrow\left(A / J_{g}\right)^{\otimes n+1}$, is a quasiisomorphism. Note also that we have not used the smoothness assumption yet.

\section{Step 5: Shapiro's Lemma and Etale Descent}

Let $\mathcal{O} \subset G$ be a conjugacy class. It is easy to see that $\bigoplus_{g \in \mathcal{O}} A_{g}^{\natural}$ and $\bigoplus_{g \in \mathcal{O}} C\left(A / J_{g}\right)$ are $G$-invariant subspaces, hence it suffices to prove the quasiisomorphism

$$
C\left(\bigoplus_{g \in \mathcal{O}} A_{g}^{\natural}\right)_{G} \rightarrow\left(\bigoplus_{g \in \mathcal{O}} C\left(A / J_{g}\right)\right)_{G}
$$

for all conjugacy classes $\mathcal{O}$. To that end, choose $g \in \mathcal{O}$ and denote by $C_{g}=\{h \in G \mid g h=h g\}$ the centralizer of $g$. Then $C_{g}$ acts on $A_{g}^{\natural}$; and on the space of coinvariants $\left(A / J_{g}\right)_{C_{g}}$ the cyclic operator $t_{n}$ satisfies $t_{n}^{n+1}=1$ therefore we obtain a mixed complex $C\left(A_{g}^{\natural}\right)_{C_{g}}$. Moreover, we have natural isomorphisms of $G$-modules

$$
\bigoplus_{g^{\prime} \in \mathcal{O}} A_{g^{\prime}}^{\natural} \simeq \operatorname{Ind} d_{g}^{G} C\left(A / J_{g}\right) ; \quad \bigoplus_{g^{\prime} \in \mathcal{O}} C\left(A / I_{g^{\prime}}\right) \simeq \operatorname{Ind}_{C_{g}}^{G} C\left(A / J_{g}\right)
$$

where $I n d_{C_{g}}^{G}$ denotes the induction map from $C_{g}$-modules to $G$-modules. By Shapiro's Lemma we are reduced to proving the quasiisomorphism

$$
C\left(A_{g}^{\natural}\right)_{C_{g}} \simeq\left(C\left(A / J_{g}\right)\right)_{C_{g}} ;
$$

which would follow once we prove that the natural surjection $A \rightarrow A / J_{g}$ induces a quasiisomorphism

$$
C\left(A_{g}^{\natural}\right)_{\langle g\rangle} \simeq C\left(A / J_{g}\right)
$$

where $\langle g\rangle \subset C_{g}$ is the cyclic subgroup generated by $g$. Note that $\langle g\rangle$ acts trivially on $A / J_{g}$ (in fact, $J_{g}$ is generated by elements $a-g(a)$ with $a \in A$ ), so we don't have to take coinvariants on the right hand side.

Now we finally use Proposition 7 (and the smoothness assumption which stands behind it). Since the properties (a), (b) and (c) are preserved by finite intersections of $G$-invariant affine open subsets, by MayerVietoris argument we can assume that there exists a point $x \in X$ and a $G_{x}$-equivariant map $\varphi: X=$ Spec $B \rightarrow T_{x}=$ Spec $A$ which is etale and satisfies (a), (b) and (c) of Proposition 7 . Let $J_{B}$ and $J_{A}$ denote the ideal of $g$-fixed points in $B$ and $A$, respectively. We have two cases:

When $g \notin G_{x}, g$ has no fixed points by (a) of , i.e. $J_{B}=B$. Then by Theorem 6 of [Lo] $C(B \rtimes\langle g\rangle)$ is quasiisomorphic to $(C(B))_{\langle g\rangle}$ (the piece obtained from the conjugacy class of identity), so the argument of Step 4 applied to $G=\langle g\rangle$ shows that $C\left(B_{g}^{\natural}\right)$ is quasiisomorphic to zero (if $g \neq 1$ ).

When $g \in G_{x}$ we use the etale map $\varphi: X \rightarrow T_{x}$ to reduce to the case of the flat space $T_{x}$. Note that until now all maps were defined in the derived category of $\Lambda$. However, it suffices to check that the map $C\left(B_{g}^{\natural}\right)_{\langle g\rangle} \rightarrow C\left(B / J_{B}\right)$ defined above is a composition of quasiisomorphisms of complexes over $k$. Note that the action $b\left(b_{0}, \ldots, b_{n}\right)=\left(b b_{0}, b_{1}, \ldots, b_{n}\right)$ actually turns both $C\left(B_{g}^{\natural}\right)$ and $C\left(B / J_{B}\right)$ into complexes of $B$-modules. We will show that they are isomorphic in the derived category of $B$ (thus, taking coinvariants in $C\left(B_{g}^{\natural}\right)_{\langle g\rangle}$ is only necessary to define an apriori mixed complex structure). 
Proposition 9 Let $X=\operatorname{Spec} B, x \in X, g \in G_{x}$ and $T_{x}=$ Spec $A$ be as above. If the map $A \rightarrow A / J_{A}$ induces a quasiisomorphism $C\left(A_{g}^{\natural}\right) \rightarrow C\left(A / J_{A}\right)$ in the derived category of $A$ then the map $B \rightarrow B / J_{B}$ induces a quasiisomorphism $C\left(B_{g}^{\natural}\right) \rightarrow C\left(B / J_{B}\right)$ in the derived category of $B$.

Proof. This proof is a minor modification of the etale descent result of [GW]. In fact, if $C\left(A_{g}^{\natural}\right) \rightarrow C\left(A / J_{A}\right)$ is a quasiisomorphism of complexes of $A$-modules then consider the following commutative diagram:

$$
\begin{array}{ccc}
B \otimes_{A} C\left(A_{g}^{\natural}\right) & \longrightarrow & B \otimes_{A} C\left(A / J_{A}\right) \simeq B / J_{B} \otimes_{A / J_{A}} C\left(A / J_{A}\right) \\
\downarrow & & \downarrow \\
C\left(B_{g}^{\natural}\right) & \longrightarrow & C\left(B / J_{B}\right)
\end{array}
$$

where the two vertical arrows are given by $b \otimes\left(a_{0}, \ldots, a_{n}\right)=\left(b a_{0}, a_{1}, \ldots, a_{n}\right)$.

The top arrow is a quasiisomorphism since $B$ is flat over $A$. The top left corner isomorphism holds since $B \simeq B / J_{B} \otimes_{A / J_{A}} A$ by part (b) of Proposition $\theta$. To show that the bottom arrow is a quasiisomorphism we will show that this property holds for the two vertical arrows. Moreover, it suffices to prove it for the left arrow only since then one can set $g=1$, replace the pair $(B, A)$ by $\left(B / J_{B}, A / J_{A}\right)$, respectively, and get the proof for the right arrow.

To prove the assertion about $B \otimes_{A} C\left(A_{g}^{\natural}\right) \rightarrow C\left(B_{g}^{\natural}\right)$ we borrow some formulas from pp. 513-514 of [BG]. Let $A_{\Delta}$ and $A_{g}$ denote bimodules over $A \otimes A$ isomorphic to $A$ as abelian groups, with the module structure given by $\left(a_{0}, a_{1}\right) \cdot a=a_{0} a a_{1}$ for $a \in A_{\Delta}$ and $\left(a_{0}, a_{1}\right) \cdot a=a_{0} a g^{-1}\left(a_{1}\right)$ for $a \in A_{g}$. Consider the free resolution $P_{\bullet}^{A}$ of $A_{\Delta}$ as $A \otimes A$-module:

$$
\ldots \rightarrow A \otimes A \otimes A \otimes A \stackrel{\partial}{\rightarrow} A \otimes A \otimes A \stackrel{\partial}{\rightarrow} A \otimes A \stackrel{\Delta}{\rightarrow} A \rightarrow 0
$$

where the $A \otimes A$-module structure on $A^{\otimes(k+2)}$ is given by

$$
\left(\bar{a}_{0}, \bar{a}_{1}\right) \cdot\left(a_{0}, \ldots, a_{k+1}\right)=\left(\bar{a}_{0} a_{0}, a_{1}, \ldots, a_{k}, a_{k+1} \bar{a}_{1}\right) ;
$$

the map $\Delta: A \otimes A \rightarrow A$ sends $\left(a_{0}, a_{1}\right)$ to $a_{0} a_{1}$ and

$$
\partial\left(a_{0}, \ldots, a_{k+1}\right)=\sum_{i=0}^{k}(-1)^{i}\left(a_{0}, \ldots, a_{i} a_{i+1}, \ldots a_{k+1}\right) .
$$

Exactness of $P_{\bullet}^{A}$ is proved using the homotopy $s:\left(a_{0}, \ldots, a_{k+1}\right) \mapsto\left(1, a_{0}, \ldots, a_{k+1}\right)$. Let $B_{g}$ and $P_{\bullet}^{B}$ be the similar objects over $B$.

Now we have a chain of isomorphisms:

$$
B \otimes_{A} C\left(A_{g}^{\natural}\right) \simeq B \otimes_{A} A_{g} \otimes_{A \otimes A} P_{\bullet}^{A} \simeq B_{g} \otimes_{A \otimes A} P_{\bullet}^{A} \simeq B_{g} \otimes_{B \otimes B}(B \otimes B) \otimes_{A \otimes A} P_{\bullet}^{A} .
$$

The first isomorphism follows from the definitions of $P_{\bullet}^{A}, C\left(A_{g}^{\natural}\right)$ and $A_{g}$. The second uses $\langle g\rangle$-equivariance of Spec $B \rightarrow$ Spec $A$ and the $A \otimes A$-module structure on $B_{g}$ which comes from $A \otimes A \rightarrow B \otimes B$. Taking into account that $C\left(B_{g}^{\natural}\right) \simeq B_{g} \otimes_{B \otimes B} P_{\bullet}^{B}$, we need to prove that the natural injective map of complexes $\rho:(B \otimes B) \otimes_{A \otimes A} P_{\bullet}^{A} \rightarrow P_{\bullet}^{B}$ becomes a quasiisomorphism after applying $B_{g} \otimes_{B \otimes B}(\ldots)$. Explicitly, we have

$$
\begin{aligned}
& \ldots \rightarrow B \otimes A \otimes B \rightarrow B \otimes B \rightarrow B \otimes_{A} B \rightarrow 0 \\
& \downarrow \rho \quad \downarrow \rho \quad \downarrow a \\
& \ldots \rightarrow B \otimes B \otimes B \rightarrow B \otimes B \rightarrow B \quad \rightarrow \quad \rightarrow \quad \rightarrow
\end{aligned}
$$

where the upper row is exact since $B \otimes B$ is flat over $A \otimes A$ and the right vertical arrow $a$ is the natural surjective map $B \otimes_{A} B \rightarrow B$.

Since $B$ is etale over $A$, the kernel $C$ of $a$ is a $B \otimes B$-module supported away from the diagonal $X_{\Delta} \subset$ $X \times X=\operatorname{Spec}(B \otimes B)$. Note that the support of $B_{g}$ coincides with the graph of the map $g^{-1}: X \rightarrow X$. We 
now claim that the supports of $B_{g}$ and $C$ are disjoint. In fact, if $\left(x_{1}, x_{2}\right) \in \operatorname{Supp}(C) \subset X \times X$ then $x_{1} \neq x_{2}$ but $\varphi\left(x_{1}\right)=\varphi\left(x_{2}\right) \in T_{x}$. If $\left(x_{0}, g^{-1}\left(x_{0}\right)\right) \in \operatorname{Supp}(C)$ then $g^{-1}$ stabilizes $\varphi\left(x_{0}\right) \in T_{x}$ but does not stabilize $x_{0} \in X$, which contradicts property (c) in Proposition $\emptyset$. Hence the supports of $B_{g}$ and $C$ are disjoint and after tensoring $B_{g} \otimes_{B \otimes B}$ the two rows in the above diagram become quasiisomorphic since $B_{g} \otimes_{B \otimes B}$ Coker $\rho$ computes $\operatorname{Tor}_{B \otimes B}^{\bullet}\left(B_{g}, C\right)=0$ (a bit more rigorously, one could first show that the localization at each maximal prime vanishes - see the last lines on p. 374 of [GW]). This finishes the proof of the proposition.

Thus, it suffices to prove the quasiisomorphism $C\left(A_{g}^{\natural}\right) \rightarrow C\left(A / J_{A}\right)$ when $A=k\left[T_{x}\right]$ is a polynomial ring with an action of the cyclic group $\langle g\rangle$ induced from its linear action on $T_{x}$.

Step 6: the Linear Case

As a last step we consider the case of a flat space $V=T_{x}=$ Spec $A$ with a linear action of the cyclic group $H=\langle g\rangle$. Let $V=V_{0} \oplus V_{1}$ where $V_{0}=V^{H}$ and $V_{1}$ is the $H$-invariant complement. Note that $\bar{A}=A / J_{A}$ is the algebra of regular functions on $V_{0}$.

Recall that for any variety $Y$, a vector bundle $E$ and its section $s$ one has a Koszul complex

$$
\ldots \rightarrow \Lambda^{3} E^{*} \stackrel{\partial}{\rightarrow} \Lambda^{2} E^{*} \stackrel{\partial}{\rightarrow} E^{*} \stackrel{\partial}{\rightarrow} \mathcal{O}_{Y} \rightarrow 0
$$

where the differential is given by contraction with $s$. We denote this $\operatorname{Koszul} \operatorname{complex}$ by $\operatorname{Kos}(Y, E, s)$. It is well-known that for a regular section $s$ and affine $Y, \operatorname{Kos}(Y, E, s)$ is a projective resolution of $\mathcal{O}_{Z}$ where $Z$ is the zero scheme of $s$.

Recall from the previous step that $C\left(A_{g}^{\natural}\right)$ is obtained by taking a particular projective resolution $P_{\bullet}^{A}$ of the diagonal copy $V_{\Delta} \subset V \times V$ and tensoring it with the $A \otimes A$-module corresponding to the graph of $g^{-1}$ : $V \rightarrow V$. Similarly, $C(\bar{A})$ is obtained by taking a particular resolution $P_{\bullet}^{\bar{A}}$ of $\left(V_{0}\right)_{\Delta} \subset V_{0} \times V_{0}$ and tensoring it with the module of functions on $\left(V_{0}\right)_{\Delta}$. As in [BG] we prove the quasiisomorphism $C\left(A_{g}^{\natural}\right) \rightarrow C(\bar{A})$ by looking at the Koszul resolutions instead of $P_{\mathbf{0}}$.

Indeed, $V_{\Delta} \subset V \times V$ is a zero scheme of a section $s$ of the trivial vector bundle with fiber $V$, given by $s\left(v_{1}, v_{2}\right)=v_{1}-v_{2}$; and similarly $\left(V_{0}\right)_{\Delta}$ is a zero scheme of the section $s_{0}=\left.s\right|_{V_{0} \times V_{0}}$ which takes values in the trivial vector bundle with the fiber $V_{0}$. Then we have a commutative diagram

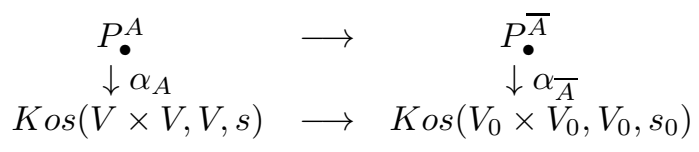

where $\alpha_{A}$ is an extension of the identity map $A_{\Delta} \rightarrow A_{\Delta}$ to the projective resolutions, and $\alpha_{\bar{A}}$ is its reduction modulo $J_{A}$ (for example, we define $\alpha$ by the formula on p. 515 of [BG]). Since $\alpha_{A}$ (resp. $\alpha_{\bar{A}}$ ) is a quasiisomorphism by definition of a projective resolution, and remains one after applying $A_{g} \otimes_{A \otimes A}$ (resp. $\bar{A}_{\Delta} \otimes_{\bar{A} \otimes \bar{A}}$ ) we only have to check that the induced map

$$
A_{g} \otimes_{A \otimes A} \operatorname{Kos}(V \times V, V, s) \rightarrow \bar{A}_{\Delta} \otimes_{\bar{A} \otimes \bar{A}} \operatorname{Kos}\left(V_{0} \times V_{0}, V_{0}, s_{0}\right)
$$

is a quasiisomorphism. But by direct computation (see [SGA], Expose VII, Proposition 2.5, for example) one can see that the left hand side is isomorphic to the left hand side tensored by $\operatorname{Kos}\left(V_{1}, V_{1}, s^{\prime}\right)$ where $s^{\prime}$ is the section of a trivial vector bundle over $V_{1}$ with fiber $V_{1}$, given by $s^{\prime}(v)=v-g^{-1}(v)$. Since $V_{1}^{H}=0$, $\operatorname{Kos}\left(V_{1}, v_{1}, s^{\prime}\right)$ is quasiisomorphic to $k$. This finishes the proof of Step 6, and the proof of Proposition 4 . 


\section{Equivalences of Derived Categories and Cohomology}

First we state a result which says that the cohomology of a complex quasiprojective variety can be recovered from an enhanced version of its derived category of vector bundles. The parts (a) and (b) of the next theorem are not stated explicitly in the papers [K1], [K2] but easily follow from their results.

Theorem 10 (a) Let $X$ be a quasiprojective variety over the field of complex numbers. In notation of Section 1 , the cyclic homology group $H C_{i}\left(\operatorname{Vect}(X), \mathbb{C}\left[u, u^{-1}\right]\right)$ is isomorphic to $H^{\text {even }}(X, \mathbb{C})$ for $i=2 k$ and $H^{\text {odd }}(X, \mathbb{C})$ for $i=2 k+1$.

(b) If $F: D^{b}(X) \rightarrow D^{b}(Y)$ is an equivalence of bounded derived categories of sheaves on two smooth projective varieties $X, Y$ over a filed $k$ then $F$ induces an isomorphism $H C \cdot(V e c t(X), W) \rightarrow H C \cdot(V e c t(Y), W)$ for any graded $k[u]$-module $W$ of finite projective dimension. In particular, if $k=\mathbb{C}$ then $F$ induces an isomorphism of complex cohomology groups.

(c) Let $X, Y$ be as in (b) and assume that a finite group $G$ acts on $X$. If char $k$ does not divide $|G|$ and there exists an equivalence of derived categories $F: D^{b}(Y) \rightarrow D_{G}^{b}(X)$ then $F$ induces an isomorphism

$$
H C \bullet(V e c t(Y), W) \simeq\left(\bigoplus_{g \in G} H C_{\bullet}\left(V e c t\left(X^{g}\right), W\right)\right)_{G}
$$

(d) If char $k=0$ (resp. $k=\mathbb{C}$ ) and $W=k\left[u, u^{-1}\right]$ then in the situation of (b) or (c) one has an isomorphism of Hodge filtrations (resp. pieces of the Hodge decomposition).

Proof. By Corollary 5.2 of [ [K2] the mixed complex $C(X)$ is quasiisomorphic to the mixed complex obtained by sheafifying the standard mixed complex of an algebra (see Section 9 of [W1]). This result holds for any field $k$. If char $k=0$ then by [FT] (in the affine case) and [W3] (general quasiprojective schemes) the homology groups $H C_{i}\left(\operatorname{Vect}(X), k\left[u, u^{-1}\right]\right.$ ) are given by the crystalline cohomology of $X$ (see Theorem 3.4 in [W3]). If particular, when $k=\mathbb{C}$ we get the Betti cohomology of $X(\mathbb{C})$. This proves (a).

To prove (b) note that by smoothness the triangulated category obtained from $\left(\mathcal{A c} c^{b}(X), \mathcal{C}^{b}(X)\right)$ is equivalent to the bounded derived category $D^{b}(X)$. By a fundamental theorem of Orlov (see Theorem 2.2, [Or] $)$ any equivalence $F$ as above is induced by a Fourier-Mukai transform with respect to some sheaf on $X \times Y$. Thus, any such equivalence $F$ automatically comes from a morphism of localization pairs and we can conclude by using the invariance property (Theorem B). Note that part (a) gives an isomorphism of complex cohomology groups only as $\mathbb{Z} / 2 \mathbb{Z}$-graded vector spaces but by the main result of [W3] this can be refined to an isomorphism of $\mathbb{Z}$-graded vector spaces as well.

Part (c) follows from Theorem 3; Theorem 1 and the fact that any equivalence $F$ still comes from a functor between localization pairs due to 8.1 and 8.2 in [K3]. Part (d) follows from [W3].

Example. Let $X-\rightarrow Y$ be an elementary flop of Bondal-Orlov, see [BO], Theorem 3.6. By loc. cit. any such flop induces an equivalence of derived categories $F: D^{b}(X) \rightarrow D^{b}(Y)$ hence an isomorphism of cohomology groups by part (b) of the above theorem. Note that the motivic integration approach only gives an equality of Betti numbers or classes in the K-theory of Hodge structures. This equivalence was extended to more general flops in dimensions 3 and 4 by Bridgeland and Namikawa.

Next we describe some situations when a derived category of sheaves on one variety is equivalent to the equivariant derived category of sheaves (with respect to a finite group action) on another variety. From now on we assume that $k=\mathbb{C}$. Let $G$ be a finite group with a unimodular action on a smooth irreducible quasiprojective variety $X$ (i.e. we require that for each $x \in X$ the image of the stabilizer $G_{x}$ in $G L\left(T_{x}\right)$ actually belongs to the subgroup $\left.S L\left(T_{x}\right)\right)$. Denote by $G H i l b(X) \subset H i l b^{|G|}(X)$ the scheme of all $G$-invariant 0-dimensional subschemes $Z \subset X$ on multiplicity $|G|$ such that $H^{0}\left(\mathcal{O}_{Z}\right)$ is isomorphic to a regular representation of $|G|$. Assume further that the generic point of $x \in X$ has trivial stabilizer and let $Y(G, X) \subset G H i l b(X)$ be the irreducible component containing all free $G$-orbits. 


\section{Theorem 11}

(1) Assume that $X, G$ and $Y=Y(G, X)$ are as above and one of the following conditions is satisfied

(a) $\operatorname{dim} X \leq 3$;

(b) $X$ is a complex symplectic variety, $G$ preserves the symplectic structure and $Y$ is a crepant resolution of $X$;

(c) $X$ is the $n$-th cartesian power of a smooth quasiprojective surface $S ; G=\Xi_{n}$ (the symmetric group) with the natural permutation action on $X$.

Then there exists an equivalence $F: D^{b}(Y) \rightarrow D_{G}^{b}(X)$ coming from a morphism of localization pairs.

(2) Assume that $\operatorname{dim} X \leq 3 ; X$ is projective and $X^{\prime}$ is another smooth projective variety with an action of a finite group $G^{\prime}$. Suppose that $X / G$ and $X^{\prime} / G^{\prime}$ are Gorenstein and there exists a common resolution of singularities $\pi$ : $Z \rightarrow X / G, \pi^{\prime}: X^{\prime} / G^{\prime}$ with $\pi^{*} K_{X} \simeq\left(\pi^{\prime}\right)^{*} K_{X^{\prime}}$. Then there exists an equivalence of categories $D_{G}^{b}(X) \simeq D_{G^{\prime}}^{b}\left(X^{\prime}\right)$. In particular, if $Z=X^{\prime}$ and $G^{\prime}=\{1\}$ (i.e. $Z$ is a crepant resolution) there exists an equivalence $D_{G}^{b}(X) \simeq D^{b}(Z)$.

Proof. Parts 1a, $1 \mathrm{~b}$ correspond to Theorem 1.2 and Corollary 1.3 in [BKR], respectively. To prove 1c, note that for $S=\mathbb{C}^{2}$ by a result of Haiman, cf. [Ha] Theorem 5.1, the variety $Y$ is isomorphic to the Hilbert scheme $H_{i l b}(S)$. Then the morphism $Y \rightarrow X / \Xi_{n}$ is semismall, see [GS], and the assertion follows from a general criterion of Theorem 1.1 in [BKR]. In general the equality $\operatorname{Hil} b^{n}(S)=Y$ is derived from the $\mathbb{C}^{2}$ case by considering completions of local rings at points $s \in S$ which are the same as for $\mathbb{C}^{2}$ since $S$ is smooth.

Part 2 is a simplified version of Theorem 1.7 due to Kawamata, cf. [Ka]. $\square$.

Corollary 12 In the first (resp. the second) case of the above theorem one has an isomorphism of the cyclic homology groups

$$
H C_{\bullet}\left(V e c t_{G}(X), W\right) \simeq H C_{\bullet}(V e c t(Y), W), \quad\left(r e s p . \quad H C_{\bullet}\left(V e c t_{G}(X), W\right) \simeq H C_{\bullet}\left(V \operatorname{lect}_{G^{\prime}}\left(X^{\prime}\right), W\right)\right)
$$

for all graded $k[u]$-modules $W$ of finite projective dimension. For $W=\mathbb{C}\left[u, u^{-1}\right]$ this reduces to isomorphisms

$$
H_{\text {orb }}^{*}(X, \mathbb{C}) \simeq H^{*}(Y, \mathbb{C}) ; \quad H_{\text {orb }}^{*}(X, \mathbb{C}) \simeq H_{\text {orb }}^{*}\left(X^{\prime}, \mathbb{C}\right)
$$

of (orbifold) cohomology groups with their Hodge filtratons.

Proof. By inspecting the proofs in [BKR] and and [Ka] one can see that all the derived equivalences above are given by functors between localization pairs. Hence the first two assertions follow from the invariance property (Theorem 3). To obtain the last pair of equalities on applies Theorem 11 and Theorem 10 (a).

Finally we note that by a recent conjecture of Kawamata, the derived equivalences 11 should be a part of more general statement. Here we formulate a part of Conjecture 1.2 in [Ka] in a slightly generalized form:

Conjecture 13 (Categorical Resolution Conjecture) Let $\mathcal{X}, \mathcal{Y}$ be smooth Deligne-Mumford stacks with Gorenstein moduli spaces $X, Y$. Suppose that there exist birational maps $f: Z \rightarrow X$ and $g: Z \rightarrow Y$ such that $f^{*}\left(K_{X}\right)=$ $g^{*}\left(K_{Y}\right)$. Then the derived categories $D^{b}(\mathcal{X})$ and $D^{b}(\mathcal{Y})$ of sheaves in the etale topology, are equivalent.

\section{Concluding Remarks}

The isomorphisms of Theorem 1 are additive counterparts of a $K$-theoretic statement in [V]. However, in loc. cit. one also has a statement concerning $K^{\prime}$-theory of singular varieties. This motivates the following conjecture:

Conjecture 14 Let $X$ be a quasiprojective scheme over a field $k, G$ a finite group acting on $X$ and assume that char $k$ does not divide $|G|$. Let $C o h(X)$ be the exact category of coherent sheaves on $X$ and $C o{ }_{G}(X)$ the exact category of $G$-equivariant sheaves. Then for any $W$ of finite projective dimension over $k[u]$ there exists an isomorphism

$$
\phi_{X}:\left(\bigoplus_{g \in G} H C_{\bullet}\left(\operatorname{Coh}\left(X^{g}\right), W\right)\right)_{G} \rightarrow H C \bullet\left(\operatorname{Coh}_{G}(X), W\right)
$$

which is functorial with respect to the (derived) pushforwards under G-equivariant proper maps. 
We expect that for smooth $X$ and $W=k\left[u, u^{-1}\right.$ (periodic cyclic homology case) this isomorphism satisfies the multiplicative part of the Cohomological Crepant Resolution Conjecture, see [Ru].

We have seen in the proof of Theorem 1 that the isomorphism $\psi_{X}$ is defined using pullbacks to the fixed point sets. We expect that, as in [V]], the isomorphism $\phi_{X}$ can be defined using direct images with respect to the closed embeddings $X^{g} \rightarrow X$. Note that $\phi_{X}$ and $\psi_{X}$ are not expected to be mutually inverse, but their composition should be invertible similarly to Lemma 4.2 and Lemma 4.3 in [V]. For singular $X$ the cyclic homology of $\operatorname{Coh}(X)$ may be different from the sheafified cyclic homology of the algebras $\mathcal{O}(U), U \subset X$; therefore the general strategy of proof has to be completely different.

Finally we state another conjecture aimed at a better understanding of the orbifold product on orbifold cohomology, see Section 3 [Ru].

Let $\mathcal{X}$ be a smooth Deligne-Mumford stack and $\pi: U \rightarrow \mathcal{X}$ be an etale cover by a scheme. Consider $Z=U \times{ }_{\pi} U \subset U \times U$. If $\pi_{i}: U \times U \times U \rightarrow U \times U$ is the projection omitting the $i$-th factor, $i=1,2,3$, then $\pi_{2}\left(\pi_{3}^{-1}(Z) \cap \pi_{1}^{-1}(Z)\right)=Z$, i.e. $Z$ is an idempotent correspondence from $U$ to itself.

In particular, we have a (finite) morphism $m: Z \times_{U} Z \rightarrow Z$ which allows to define for any two objects $\mathcal{F}, \mathcal{G} \in D^{b}(Z)$ a third object

$$
\mathcal{F} * \mathcal{G}:=m_{*}\left(p_{1}^{*} \mathcal{F} \otimes p_{2}^{*} \mathcal{G}\right)
$$

where $p_{1}, p_{2}$ are the projections of $Z \times_{U} Z$ to $Z$, and all operations are understood in the derived sense.

The $*$-product in general is not commutative, but it is associative (more precisely, there is a canonical isomorphism between $\mathcal{F} *(\mathcal{G} * \mathcal{H})$ and $(\mathcal{F} * \mathcal{G}) * \mathcal{H})$. When $\mathcal{X}$ is the quotient stack $[X / G]$ we can take $U=X$ and then $Z=\coprod_{g \in G} X^{g}$ while $m: Z \times_{U} Z \rightarrow Z$ is given by pointwise group product within stabilizers.

Conjecture 15 When $\mathcal{X}=[X / G]$ and $U=X$ the above product $(\mathcal{F}, \mathcal{G}) \mapsto \mathcal{F} * \mathcal{G}$ induces the product of the periodic cyclic homology of $Z=\coprod_{g \in G} X^{g}$ which coincides with the product on $A(X, G)=H^{*}(Z, \mathbb{C})$ described in Section 3 of [Ru]. Thus, taking the (co)invariants with respect to G-action gives the orbifold product structure on $H_{\text {orb }}^{*}(X / G, \mathbb{C})$.

Note that the only place where smoothness of $\mathcal{X}$ is important is the relation between cyclic homology of coherent sheaves and the orbifold cohomology groups. However, we could define orbifold cohomology for singular Deligne-Mumford stacks via cyclic homology of coherent sheaves; such a definition, perhaps, would give invariants which behave better than those defined via the usual cohomology.

\section{References}

[AK] Akbarpour, R.; Khalkhali, M.: Hopf Algebra Equivariant Cyclic Homology and Cyclic Homology of Crossed Product Algebras, preprint math.KT/0011248

[B] Burghelea, D.: The cyclic homology of the group rings. Comment. Math. Helv. 60 (1985), no. 3, 354365.

[BG] Block, J.; Getzler, E.: Equivariant cyclic homology and equivariant differential forms. Ann. Sci. École Norm. Sup. (4) 27 (1994), no. 4, 493-527.

[BKR] Bridgeland, T.; King, A.; Reid, M.: The McKay correspondence as an equivalence of derived categories. J. Amer. Math. Soc. 14 (2001), no. 3, 535-554.

[BL] Bernstein, J.; Lunts, V.: Equivariant sheaves and functors. Lecture Notes in Mathematics, 1578. Springer-Verlag, Berlin, 1994.

[BN] Brylinski, J.-L.; Nistor, V.: Cyclic cohomology of étale groupoids. K-Theory 8 (1994), no. 4, 341-365.

[BO] Bondal A., Orlov D.: Semiorthogonal decomposition for algebraic varieties, preprint alg-geom/9506012. 
[Br] Brylinski, J.-L.: Cyclic homology and equivariant theories. Ann. Inst. Fourier (Grenoble) 37 (1987), no. $4,15-28$.

[BR] Bardsley, P.; Richardson, R. W.: Étale slices for algebraic transformation groups in characteristic $p$. Proc. London Math. Soc. (3) 51 (1985), no. 2, 295-317.

[Cr] Crainic, M.: Cyclic cohomology of étale groupoids: the general case. K-Theory 17 (1999), no. 4, 319362.

[FT] Feigin, B.L.; Tsygan, B.L.: Additive $K$-theory. $K$-theory, arithmetic and geometry (Moscow, 1984-1986), 67-209, Lecture Notes in Math., 1289, Springer, Berlin, 1987.

[GJ1] Getzler, E.; Jones, J. D. S.: $A_{\infty}$-algebras and the syclic bar complex Illinois J. of Math. 34 (1990), no. 2, 256-283.

[GJ2] Getzler, E.; Jones, J. D. S.: The cyclic homology of crossed product algebras. J. Reine Angew. Math. 445 (1993), 161-174.

[GS] Göttsche, L.; Soergel, W.: Perverse sheaves and the cohomology of Hilbert schemes of smooth algebraic surfaces. Math. Ann. 296 (1993), no. 2, 235-245.

[GW] Geller, S.; Weibel, C.: Étale descent for Hochschild and cyclic homology. Comment. Math. Helv. 66 (1991), no. 3, 368-388.

[Ha] Haiman, M.: Hilbert schemes, polygraphs and the Macdonald positivity conjecture. J. Amer. Math. Soc. 14 (2001), no. 4, 941-1006.

[HH] Hirzebruch, F.; Höfer, T.: On the Euler number of an orbifold. Math. Ann. 286 (1990), no. 1-3, 255-260.

[Ka] Kawamata, Y.: D-equivalence and K-equivalence, preprint math.AG/0205287.

[K1] Keller, B: On the cyclic homology of exact categories, J. Pure Appl. Algebra 136 (1999) no. 1, 1-56.

[K2] Keller, B.: On the cyclic homology of ringed spaces and schemes. Doc. Math. 3 (1998), 231-259.

[K3] Keller, B.: Deriving DG categories. Ann. Sci. École Norm. Sup. (4) 27 (1994), no. 1, 63-102.

[Lo] Lorenz, M.: On Galois descent for Hochschild and cyclic homology. Comment. Math. Helv. 69 (1994), no. $3,474-482$.

[N] Nistor, V.: Cyclic cohomology of crossed products by algebraic groups. Invent. Math. 112 (1993), no. $3,615-638$.

[Or] Orlov D.: Equivalences of derived categories and K3 surfaces. Algebraic geometry, 7. J. Math. Sci. (New York) 84 (1997), no. 5, 1361-1381; also preprint alg-geom/9606006.

[Ru] Ruan, Y.: Stringy Orbifolds, preprint math.AG/0201123.

[SGA] SGA 6: Théorie des intersections et théoréme de Riemann-Roch. Séminaire de Géométrie Algébrique du Bois-Marie 1966-1967. LNM 225. Springer-Verlag, Berlin-New York, 1971.

[To] Toen, B.: Riemann-Roch Theorems for Deligne-Mumford Stacks, preprint math.AG/9803076.

[TT] Thomason, R. W.; Trobaugh, T.: Higher algebraic $K$-theory of schemes and of derived categories. The Grothendieck Festschrift, Vol. III, 247-435, Progr. Math., 88, Birkhäuser Boston, Boston, MA, 1990.

[V] Vistoli, A.: Higher equivariant K-theory for finite group actions. Duke Math. J. 63 (1991), no. 2, 399419. 
[W1] Weibel, C.: An introduction to homological algebra. Cambridge Studies in Advanced Mathematics, 38. Cambridge University Press, Cambridge, 1994.

[W2] Weibel, C.: Cyclic homology for schemes. Proc. Amer. Math. Soc. 124 (1996), no. 6, 1655-1662.

[W3] Weibel, C.: The Hodge filtration and cyclic homology. K-Theory 12 (1997), no. 2, 145-164.

[Y] Yasuda, T.: Twisted jet, motivic measure and orbifold cohomology, preprint math.AG/0110228.

Department of Mathematics

253-37 Caltech

Pasadena, CA 91125, USA

email: baranovs@caltech.edu 